\title{
First Results from the HI Parkes Zone of Avoidance Survey
}

\author{
P. A. Henning \\ Institute for Astrophysics, University of New Mexico, Albuquerque, NM, \\ 87131
}

L. Staveley-Smith

Australia Telescope National Facility, CSIRO, P.O. Box 76, Epping, NSW 1710, Australia

R. C. Kraan-Korteweg

Departamento de Astronomia, Universidad de Guanajuato, Guanajuato, Gto. CP 36000, Mexico

E. M. Sadler

School of Physics, University of Sydney, NSW 2006, Australia

\begin{abstract}
The HI Parkes Zone of Avoidance Survey is a $21 \mathrm{~cm}$ blind search with the multibeam receiver on the $64-m$ radiotelescope, looking for galaxies hidden behind the southern Milky Way. The first phase of the survey has uncovered 107 galaxies, two-thirds of which were previously unknown. The addition of these galaxies to existing extragalactic catalogs allows the connectivity of large-scale structures across the Zone of Avoidance within $3500 \mathrm{~km} \mathrm{~s}^{-1}$ to become evident. No nearby hidden "Andromeda-class" object was uncovered. Our census of the most dynamically important $\mathrm{HI}$-bearing nearby galaxies is now complete, at least for those objects whose HI profiles are not totally buried in the Galactic HI signal. The full survey is ongoing, and is expected to produce a catalog of thousands of objects when it is finished.
\end{abstract}

\section{Introduction}

The dust and high stellar density of the Milky Way obscures up to $25 \%$ of the optical extragalactic sky, creating a Zone of Avoidance ( $\mathrm{ZOA}$ ). The resulting incomplete coverage of surveys of external galaxies leaves open the possibility that dynamically important structures, or even nearby massive galaxies, remain undiscovered.

Careful searches in the optical and infrared wave bands can narrow the ZOA, (see Kraan-Korteweg, this volume) but in the regions of highest obscuration and infrared confusion, only radio surveys can find galaxies. The $21 \mathrm{~cm}$ line of neutral hydrogen (HI) passes readily through the obscuration, so galaxies with 
sufficient HI can be found through detection of their $21 \mathrm{~cm}$ emission. Of course, this method will miss HI-poor, early-type galaxies, and cannot discriminate HI galaxies with redshifts near zero velocity from Galactic HI.

Here we describe an HI blind survey for galaxies in the southern ZOA conducted with the new multibeam receiver on the $64-\mathrm{m}$ Parkes telescope. A survey of $\mathrm{HI}$ galaxies in the northern $\mathrm{ZOA}$ is underway with the Dwingeloo radiotelescope (Henning et al. 1998; Rivers et al. this volume).

\section{The Shallow Survey Observations and Data Analysis}

The HI Parkes ZOA survey covers the southern ZOA $\left(212^{\circ} \leq l \leq 36^{\circ} ;|b| \leq 5^{\circ}\right)$ over the velocity range $(\mathrm{cz})=-1200$ to $12700 \mathrm{~km} \mathrm{~s}^{-1}$. The multibeam receiver is a focal plane array with 13 beams arranged in an hexagonal grid. The spacing between adjacent beams is about two beamwidths, each beamwidth being 14 arcmin. The survey is comprised of 23 contiguous rectangular fields which are scanned parallel to the galactic equator. Eventually, each patch will be observed 25 times, with scans offset by about 1.5 arcmin in latitude. The shallow survey discussed here consists of two scans in longitude separated by $\Delta b=17$ arcmin, resulting in an rms noise of about $15 \mathrm{mJy}$, equivalent to a $5 \sigma$ HI mass detection limit of $4 \times 10^{6} \mathrm{~d}_{\mathrm{Mpc}}^{2} \mathrm{M}_{\odot}$ (for a galaxy with the typical linewidth of $200 \mathrm{~km} \mathrm{~s}^{-1}$ ).

After calibration, baseline-subtraction, and creation of data cubes, all done with specially developed routines based on aips++ (Barnes et al. 1998, Barnes 1998) the data are examined by eye using the visualization package Karma (http://www.atnf.csiro.au/karma/). The data are first displayed as right ascension - velocity planes, in strips of constant declination. Data cubes are then rotated, and right ascension - declination planes are checked for any suspected galaxies (eg. Figure 1).

\section{Galaxies Found by the Shallow Survey}

The shallow 21-cm survey of the southern ZOA has been completed, and 107 galaxies with peak HI flux densities $\geq$ about $80 \mathrm{mJy}$ have been cataloged. Refinement of the measurement of their HI characteristics is ongoing, but the objects seem to be normal galaxies. Most of the galaxies are within $4000 \mathrm{~km} \mathrm{~s}^{-1}$, which is about the redshift limit for detection of normal spirals of this shallow phase of the survey. As the deep survey continues, spirals at higher velocities will be recovered. Of the 107 objects, 28 have counterparts in the NASA/IPAC Extragalactic Database (NED) with matching positions and redshifts. Optical absorption, estimated from the Galactic dust data of Schlegel et al. (1998), ranges from $A_{B}=1$ to more than 60 mag at the positions of the 107 galaxies, and is patchy over the survey area. No objects lying behind more than about 6 mag of obscuration have confirmed counterparts in NED, as expected.

The connection of these objects with known large-scale structures in optically unobscured regions is discussed by Kraan-Korteweg in this volume, and the reader is referred to her paper for graphical depiction. The shallow multibeam HI survey connects structures all the way across the ZOA within $\mathrm{v}<3500 \mathrm{~km} \mathrm{~s}^{-1}$. The ongoing, deep ZOA survey will have sufficient sensitivity to connect structures at higher redshifts. 

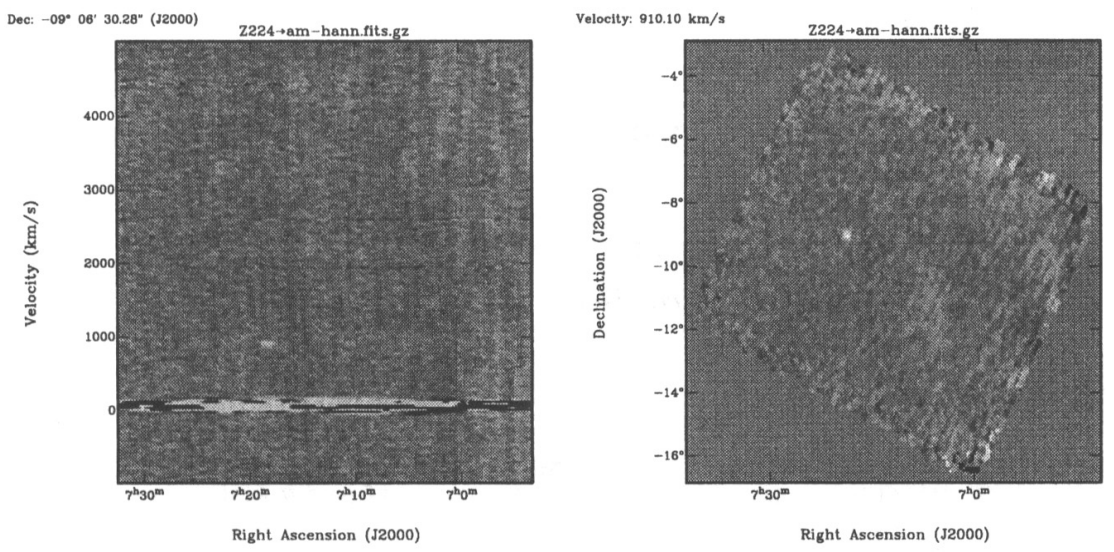

Figure 1. Left panel shows a right ascension - velocity slice to $5000 \mathrm{~km} \mathrm{~s}^{-1}$ which includes a galaxy discovered by the survey. Galactic HI appears as the strong horizontal feature at zero velocity. Note the extragalactic HI signal at $7^{\mathrm{h}} 18^{\mathrm{m}}, 900 \mathrm{~km} \mathrm{~s}^{-1}$. Right panel shows the right ascension - declination plane at the velocity of the suspected signal. The galaxy is evident at $7^{\mathrm{h}} 18^{\mathrm{m}},-9^{\circ}$

While 14 of the 107 galaxies lie within $1000 \mathrm{~km} \mathrm{~s}^{-1}$ and are therefore fairly nearby, all of the newly-discovered objects have peak HI flux densities an order of magnitude or more lower than the Circinus galaxy . Thus, it seems our census of the most dynamically important, HI-bearing nearby galaxies is now complete, at least for those objects with velocities offset from Galactic HI. Simulations are currently being conducted to investigate our sensitivity to HI galaxies whose signals lie within the frequency range of the Milky Way's HI.

Acknowledgments. We thank HIPASS ZOA collaborators R. D. Ekers, A. J. Green, R. F. Haynes, S. Juraszek, M. J. Kesteven, B. S. Koribalski, R. M. Price, and A. Schröder. This research has made use of the NASA/IPAC Extragalactic Database (NED) which is operated by the Jet Propulsion Laboratory, Caltech, under contract with the National Aeronautics and Space Administration. The research of P.H. is supported by NSF Faculty Early Career Development (CAREER) Program award AST 95-02268.

\section{References}

Barnes, D.G. 1998, in ADASS VII, eds. Albrecht, R., Hook, R.N., \& Bushouse, H.A., San Francisco: ASP

Barnes, D.G., Staveley-Smith, L, Ye, T., \& Osterloo, T. 1998, in ADASS VII, eds. Albrecht, R., Hook, R.N., \& Bushouse, H.A., San Francisco: ASP

Henning, P.A., Kraan-Korteweg, R.C., Rivers, A.J., Loan, A.J., Lahav, O., \& Burton, W.B. 1998, AJ, 115, 584

Schlegel, D.J., Finkbeiner, D.P., \& Davis, M. 1998, ApJ, 500, 525 